\title{
Improving Shape Depiction under Arbitrary Rendering
}

\author{
Romain Vergne, Romain Pacanowski, Pascal Barla, Xavier Granier, Christophe Schlick
}

\begin{abstract}
Based on the observation that shading conveys shape information through intensity gradients, we present a new technique called Radiance Scaling that modifies the classical shading equations to offer versatile shape depiction functionalities. It works by scaling reflected light intensities depending on both surface curvature and material characteristics. As a result, diffuse shading or highlight variations become correlated to surface feature variations, enhancing concavities and convexities. The first advantage of such an approach is that it produces satisfying results with any kind of material for direct and global illumination: we demonstrate results obtained with Phong and AshikminShirley BRDFs, Cartoon shading, sub-Lambertian materials, perfectly reflective or refractive objects. Another advantage is that there is no restriction to the choice of lighting environment: it works with a single light, area lights, and inter-reflections. Third, it may be adapted to enhance surface shape through the use of precomputed radiance data such as Ambient Occlusion, Prefiltered Environment Maps or Lit Spheres. Finally, our approach works in real-time on modern graphics hardware making it suitable for any interactive 3D visualization.
\end{abstract}

Index Terms-Expressive rendering, NPR, shape depiction, shading, global illumination.

\section{INTRODUCTION}

$\mathrm{T}$ HE depiction of object shape has been a subject of increased interest in the Computer Graphics community since the work of Saito and Takahashi [1]. Inspired by their pioneering approach, many rendering techniques have focused on finding an appropriate set of lines to depict object shape. In contrast to line-based approaches, other techniques depict object shape through shading. Maybe the most widely used of these is Ambient Occlusion [2], which measures the occlusion of nearby geometry. Both types of techniques make drastic choices for the type of material, illumination and style: line-based approaches often ignore material and illumination and depict mainly sharp surface features, whereas occlusion-based techniques convey deep cavities for diffuse objects under ambient illumination.

More versatile shape enhancement techniques are required to accommodate the needs of modern Computer Graphics applications. They should work with realistic as well as stylized rendering to adapt to the look-and-feel of a particular movie or video game production. A wide variety of materials should be taken into account, such as diffuse, glossy and transparent materials, with specific controls for each material component. A satisfying method should work for various illumination settings ranging from complex illumination for movie production, to simple or even precomputed illumination for video games. On top of these requirements, enhancement methods should be fast enough to be incorporated in interactive applications or to provide instant feedback for previewing.

- R. Vergne, P. Barla, X.Granier and C.Schlick are affiliated with the INRIA Bordeaux University, 351 cours de la Libration, 33405 Talence, France.E-mail: \{vergne,barla,granier,schlick\}@labri.fr

- R. Pacanowski is affiliated with the CEA-CESTA, BP 2 - 33114 Le Barp, France. E-mail: romain.pacanowski@cea.fr

- X. Granier is also affiliated with State Key Lab of CAD\&CG, Zhejiang University, 388 Yuhangtang Road, Hangzhou, 310058 China.
This versatility has been recently tackled by techniques that either modify the final evaluation of reflected radiance as in 3D Unsharp masking [3], or modify it for each incoming light direction as in Light Warping [4]. These techniques have shown compelling enhancement abilities without relying on any particular style, material or illumination constraint. Unfortunately, as detailed in Section 2, they provide at best a partial control on the enhancement process and produce unsatisfying results or even artifacts for specific choices of material or illumination. Moreover, both methods are dependent on scene complexity: 3D Unsharp Masking performances slow down with an increasing number of visible vertices, whereas Light Warping requires a dense sampling of the environment illumination, with a non-negligible overhead per light ray.

This paper gives an extended description of the Radiance Scaling technique [5]. The main contribution of Radiance Scaling is to depict shape through shading in a way that combines the advantages of 3D Unsharp Masking and Light Warping while providing a more versatile and faster solution. The key idea is to adjust reflected light intensities in a way that depends on both surface curvature and material characteristics, as explained in Section 3. As with 3D Unsharp Masking, enhancement is performed by introducing variations in reflected light intensity, an approach that works for any kind of illumination. However, this is not performed indiscriminately at every surface point and for the outgoing radiance only, but in a curvature-dependent manner and for each incoming light direction as in Light Warping. The main tool to achieve this enhancement is a novel scaling function presented in Section 4. In addition, Radiance Scaling takes material characteristics into account, which makes the method easy to adapt to different rendering scenarios as shown in Section 5. Comparisons with related techniques and directions for future work are given in Section 6. 


\section{Previous work}

Most of the work done for the depiction of shape in Computer Graphics concerns line-based rendering techniques. Since the seminal work of Saito and Takahashi [1], many novel methods (e.g.,[6], [7], [8], [9], [10], [11], [12], [13]) have been proposed. Most of these techniques focus on depicting shape features directly, and thus make relatively little use of material or illumination information, with the notable exception of Lee et al. [10].

A number of shading-based approaches have also shown interesting abilities for shape depiction. The most widely used of these techniques is Ambient Occlusion [2], which measures the occlusion of nearby geometry. The method rather tends to depict deep cavities, whereas shallow (yet salient) surface details are often missed or even smoothed out. Moreover, enhancement only occurs implicitly (there is no control over depicted features), and the method is limited to diffuse materials and ambient lighting. It is also related to Accessibility shading techniques (e.g., [14]), which convey information about concavities of a $3 \mathrm{D}$ object.

The recent $3 D$ Unsharp Masking technique of Ritshel et al. [3] addresses limitations on the type of material or illumination. It consists in applying the Cornsweet Illusion effect [15] to outgoing radiance on an object surface. The approach provides interesting enhancement not only with diffuse materials, but also with glossy objects, shadows and textures. However, the method is applied indiscriminately to all these effects, and thus enhances surface features only implicitly, when radiance happens to be correlated with surface shape. Moreover, it produces artifacts when applied to glossy objects: material appearance is then strongly altered and objects tend to look sharper than they really are. Hence, the method is likely to create noticeable artifacts when applied to highly reflective or refractive materials.

In this paper, we rather seek a technique that enhances object shape explicitly, with intuitive controls for the user. Previous methods [16], [17], [18], [19], [4] differ in the geometric features they enhance and on the constraints they put on materials, illumination or style. For instance, Cignoni et al. [17] directly modify the normal field by scaling up its high-frequency component. Exaggerated Shading [18] makes use of normals at multiple scales to define surface relief and relies on a Half-Lambertian to depict relief at grazing angles. The two techniques of Vergne et al. [19], [4] make use of a view-centered curvature tensor to define surface features. These features are enhanced by specific NPR styles in Apparent Relief [19]. Their more recent and general Light Warping technique [4] improves the viewcentered curvature tensor and enhances surface features by locally stretching or compressing reflected light patterns around the view direction.

Although this technique puts no constraint on the choice of material or illumination, its effectiveness decreases with lighting environments that do not exhibit natural statistics. It also requires a dense sampling of illumination, and is thus not adapted to simplified lighting such as found in video games, or to the use of precomputed radiance methods. Moreover, highly reflective or refractive materials produce complex warped patterns that tend to make rendering less legible. The authors partly compensate for their limitation of environments with natural statistics by performing a curvature-dependent intensity adjustment, an approach very similar to Mean Curvature Shading [16]. Radiance Scaling takes this idea further by adjusting reflected light intensity for each incoming light direction.

\section{Overview}

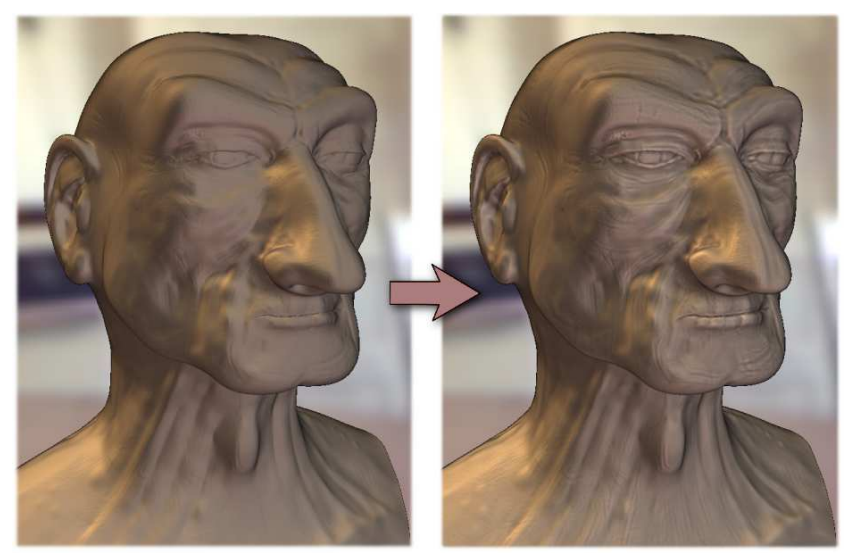

Fig. 1: Our novel Radiance Scaling technique enhances surface features through shading, here only making use of specularities. Observe how various surface details at various scales are enhanced around the eyes, the mouth and the neck for instance.

The key observation of this paper is that explicitly correlating reflected lighting variations to surface feature variations leads to an improved depiction of object shape. For example, consider a highlight reflected from a glossy object; by increasing reflected light intensity in convex regions and decreasing it in concave ones, the highlight looks as if it were attracted toward convexities and repelled from concavities (see Figure 1). Such an adjustment improves the distinction between concave and convex surface features, and does not only take surface features into account, but also material characteristics. Indeed, reflected light intensity has an altogether different distribution across the surface depending on whether the material is glossy or diffuse.

The main idea of Radiance Scaling is thus to adjust reflected light intensity per incoming light direction in a way that depends on both surface curvature and material characteristics. Formally, we rewrite the reflected radiance equation as follows:

$$
L^{\prime}(\mathbf{p} \rightarrow \mathbf{e})=\int_{\Omega} \rho(\mathbf{e}, \boldsymbol{\ell})(\mathbf{n} \cdot \boldsymbol{\ell}) \sigma(\mathbf{p}, \mathbf{e}, \boldsymbol{\ell}) L(\mathbf{p} \leftarrow \boldsymbol{\ell}) d \boldsymbol{\ell}
$$

where $L^{\prime}$ is the enhanced radiance, $\mathbf{p}$ is a surface point, $\mathbf{e}$ is the direction toward the eye, $\mathbf{n}$ is the surface normal at $\mathbf{p}, \Omega$ is the hemisphere of directions around $\mathbf{n}, \boldsymbol{\ell}$ is a light 
direction, $\rho$ is the material BRDF, $\sigma$ is a scaling function and $L$ is the incoming radiance.

The scaling function is a short notation for $\sigma_{\alpha, \gamma}(\kappa(\mathbf{p}), \delta(\mathbf{e}, \ell))$. The curvature mapping function $\kappa(\mathbf{p}): \mathbb{R}^{3} \rightarrow[-1,1]$ computes normalized curvature, where -1 corresponds to maximum concavities, 0 to planar regions and 1 to maximum convexities. The reflectance mapping function $\delta(\mathbf{e}, \boldsymbol{\ell}): \Omega^{2} \rightarrow[0,1]$ computes normalized values, where 0 corresponds to minimum reflected intensity, and 1 to maximum reflected intensity. Intuitivelly, it identifies the light direction that contributes the most to reflected intensity.

We describe the formula for the scaling function $\sigma$ and the choice of curvature mapping function $\kappa$ in Section 4. We then show how Radiance Scaling is adapted to various rendering scenarios by a proper choice of reflectance mapping function $\delta$ in Section 5 .

\section{Scaling function}

The goal of the scaling function $\sigma$ is to map a curvature measure $\kappa$ and a reflectance measure $\delta$ to the scaling term in Equation 1. It is tailored to enhance existing surface features, which we translate into three properties. First, it is required to be monotonic so that no new shading extremum is created. Second, when no surface feature is found (i.e., in planar surface regions), the function must have no influence on reflected lighting, and hence in this case $\sigma=1$. Third, the way surface features are either darkened $(\sigma<1)$ or brightened $(\sigma>1)$ should be easily controlled via a single parameter. The following function fulfills these requirements, as seen in Figure 2:

$$
\sigma_{\alpha, \gamma}(\kappa, \delta)=\frac{\alpha e^{\gamma \kappa}+\delta\left(1-\alpha\left(1+e^{\gamma \kappa}\right)\right)}{\alpha+\delta\left(e^{\gamma \kappa}-\alpha\left(1+e^{\gamma \kappa}\right)\right)}
$$

where $\alpha \in(0,1)$ controls the location of the scalinginvariant point of $\sigma$ and $\gamma \in[0, \infty)$ is the scaling magnitude. The scaling-invariant point controls how variations in shading depict surface feature variations. For convex features, reflected lighting intensities above $\alpha$ are brightened and those below $\alpha$ are darkened. For concave features, the opposite effect is obtained. Various choices for $\alpha$ are illustrated in Figure 3.
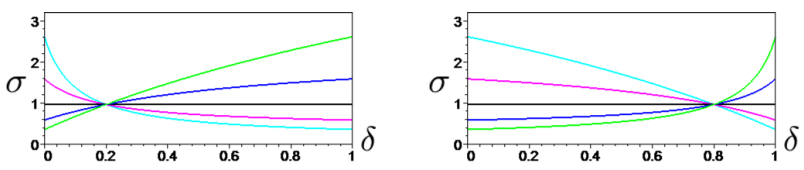

Fig. 2: Two plots of a set of scaling functions with different scaling-invariant points (left: $\alpha=0.2$; right: $\alpha=0.8$ ), and using increasing curvatures $\kappa=\{-1,-1 / 2,0,1 / 2,1\}$.

Equation 2 has a number of interesting properties, as can be seen in Figure 2. First note that the function is equal to 1 only at $\delta=\alpha$ or when $\kappa=0$ as required. Second, concave and convex features have a reciprocal effect on

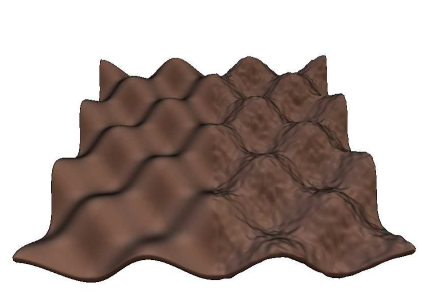

(a)

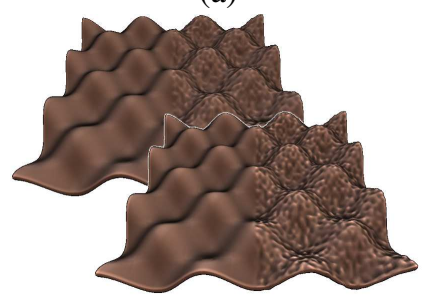

(c) (b)

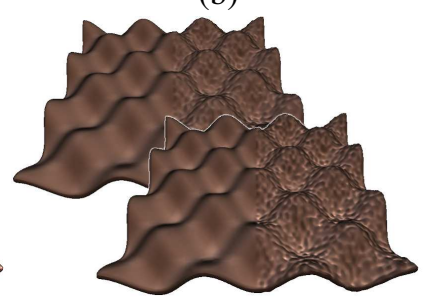

(d)

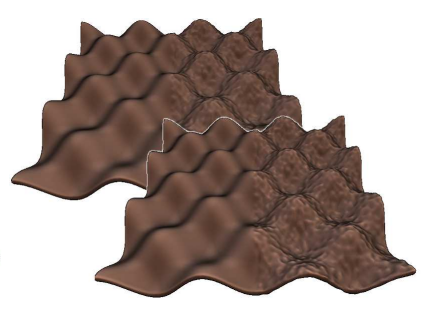

Fig. 3: Scaling parameters (a) The original rendering with no scaling $(\gamma=0)$. ( $b-d)$ Our enhanced result using two scaling coefficients (top: $\gamma=10$; bottom: $\gamma=20$ ) and three scaling-invariant points: $(b)$ with $\alpha=0.5$, there is a good equilibrium between concavity and convexity enhancement; (c) with $\alpha=0.01$, convexities are more brightened; $(d)$ with $\alpha=0.99$, shading gradients become reversed.

the scaling function: $\sigma_{\alpha, \gamma}(\kappa, \delta)=1 / \sigma_{\alpha, \gamma}(-\kappa, \delta)$. A third property is that the function is symmetric with respect to $\alpha: \sigma_{\alpha, \gamma}(\kappa, 1-\delta)=1 / \sigma_{1-\alpha, \gamma}(\kappa, \delta)$. These choices make the manipulation of the scaling function comprehensible for the user, as illustrated in Figure 3.

Our choice for the curvature mapping function $\kappa$ is based on the view-centered curvature tensor of Vergne et al. [4]. We first compute a relative depth gradient $\mathbf{g}$ at a point $\mathbf{p}$ from its normal $\mathbf{n}=\left(n_{x}, n_{y}, n_{z}\right)$ expressed in camera space:

$$
\mathbf{g}(\mathbf{p})=\left(\begin{array}{c}
-n_{x} / n_{z}^{f} \\
-n_{y} / n_{z}^{f}
\end{array}\right)
$$

where we have introduced a foreshortening parameter $f \in$ $[0, \infty)$. This new parameter serves to control whether surfaces turning away from the view direction are considered more or less curved. The Hessian of the depth field is then computed by differentiating the gradient:

$$
\mathbf{H}(\mathbf{p})=\nabla^{T} \mathbf{g}(\mathbf{p})=\left(\begin{array}{ll}
\mathbf{g}_{x} & \mathbf{g}_{y}
\end{array}\right)
$$

where $\mathbf{g}_{x}$ and $\mathbf{g}_{y}$ are the first-order derivatives of $\mathbf{g}$ in the $\mathbf{x}$ and $\mathbf{y}$ directions.

In the general case, we employ an isotropic curvature mapping: mean curvature is mapped to the $[-1,1]$ range via $\kappa(\mathbf{p})=\tanh \left(\kappa_{u}+\kappa_{v}\right)$ where $\kappa_{u}$ and $\kappa_{v}$ are the principal curvatures of $\mathbf{H}(\mathbf{p})$. Figure 4 compares mean curvature displays obtained from $\mathbf{H}$ with different values of the foreshortening parameter $f$, and with a conventional objectspace computation as well. Other object-space measures could have been used (e.g., [20]). 


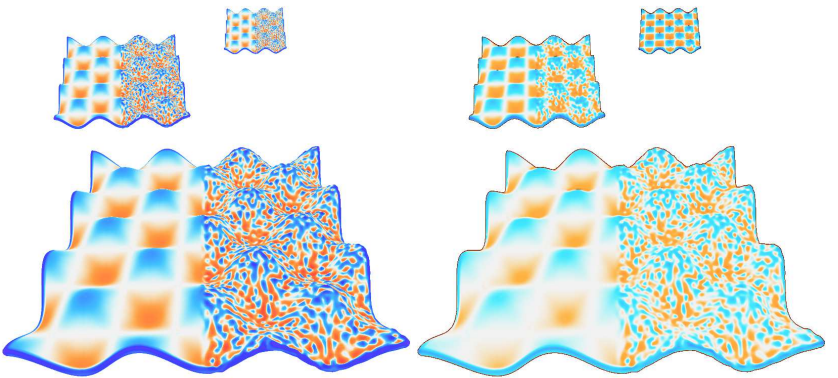

(a)

(b)

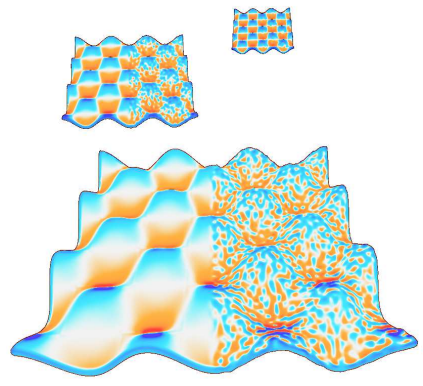

(c)
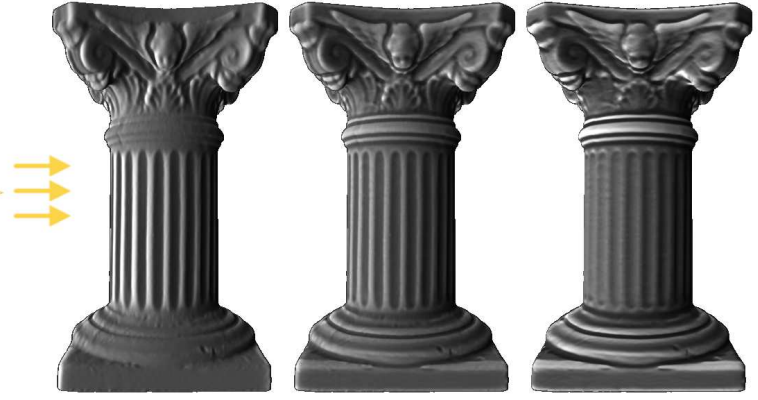

Fig. 5: Scaling anisotropy. With the light direction coming from the left, we show the effect of the anisotropy parameter $\lambda$. From left to right, we show anisotropy orthogonal to light direction $(\lambda=-1)$, isotropy $(\lambda=0)$ and anisotropy in the direction of the light $(\lambda=1)$. Observe how different features are enhanced with different values of $\lambda$.

\subsection{Simple lighting with Phong shading model}

In interactive applications such as video games, it is common to make use of simple shading models such as Phong shading, with a restricted number of light sources. Radiance Scaling allows users to control each term of Phong's shading model independently, as explained in the following.

With a single light source and Phong shading, Equation 1 becomes

$$
L^{\prime}(\mathbf{p} \rightarrow \mathbf{e})=\sum_{j} \rho_{j}\left(\mathbf{e}, \boldsymbol{\ell}_{0}\right) \sigma_{j}\left(\mathbf{p}, \mathbf{e}, \boldsymbol{\ell}_{0}\right) L_{j}\left(\boldsymbol{\ell}_{0}\right)
$$

For more advanced control though, we provide an anisotropic curvature mapping, whereby $\kappa$ is defined as a function of $\ell$ as well:

$$
\kappa(\mathbf{p}, \boldsymbol{\ell})=\tanh \left(\left(H+\lambda \Delta_{\kappa}\right) \ell_{u}^{2}+\left(H-\lambda \Delta_{\kappa}\right) \ell_{v}^{2}+H \ell_{z}^{2}\right)
$$

with the light direction $\ell=\left(\ell_{u}, \ell_{v}, \ell_{z}\right)$ expressed in the $(\mathbf{u}, \mathbf{v}, \mathbf{z})$ reference frame, where $\mathbf{u}$ and $\mathbf{v}$ are the principal directions of $\mathbf{H}$ and $\mathbf{z}$ is the direction orthogonal to the picture plane. $H=\kappa_{u}+\kappa_{v}$ corresponds to mean curvature and $\Delta_{\kappa}=\kappa_{u}-\kappa_{v}$ is a measure of curvature anisotropy. Intuitively, the function outputs a curvature value that is obtained by linearly blending principal and mean curvatures based on the projection of $\ell$ in the picture plane. The parameter $\lambda \in[-1,1]$ controls the way anisotropy is taken into account (see Figure 5): when $\lambda=0$, scaling is isotropic $(\forall \boldsymbol{\ell}, \kappa(\mathbf{p}, \boldsymbol{\ell})=\tanh (H))$; when $\lambda=1$, scaling is anisotropic (e.g., $\kappa(\mathbf{p}, \mathbf{u})=\tanh \left(\kappa_{u}\right)$ ); and when $\lambda=-1$, scaling is anisotropic, but directions are reversed (e.g., $\left.\kappa(\mathbf{p}, \mathbf{u})=\tanh \left(\kappa_{v}\right)\right)$. When $\boldsymbol{\ell}$ is aligned with $\mathbf{z}$, only isotropic scaling may be applied.

\section{Rendering scenarios}

We now explain how the choice of reflectance mapping function $\delta$ permits the enhancement of surface features in a variety of rendering scenarios. Reported performances have been measured at a $800 \times 600$ resolution using a NVIDIA Geforce 8800 GTX. where $j \in\{a, d, s\}$ iterates over the ambient, diffuse and specular components of Phong's shading model and $\boldsymbol{\ell}_{0}$ is the light source direction at point p. For each component, $L_{j}$ corresponds to light intensity ( $L_{a}$ is a constant). The ambient, diffuse and specular components are given by $\rho_{a}=1, \rho_{d}\left(\boldsymbol{\ell}_{0}\right)=\left(\mathbf{n} \cdot \boldsymbol{\ell}_{0}\right)$ and $\rho_{s}\left(\mathbf{e}, \boldsymbol{\ell}_{0}\right)=\left(\mathbf{r} \cdot \boldsymbol{\ell}_{0}\right)^{\eta}$ respectively, with $\mathbf{r}=2(\mathbf{n} \cdot \mathbf{e})-\mathbf{e}$ the mirror view direction and $\eta \in[0, \infty)$ a shininess parameter.

The main difference between shading terms resides in the choice of reflectance mapping function. Since Phong lobes are defined in the $[0,1]$ range, the most natural choice is to use them directly as mapping functions: $\delta_{j}=\rho_{j}$. It not only identifies a reference direction in which reflected light intensity will be maximal (e.g., $\mathbf{n}$ for $\delta_{d}$ or $\mathbf{r}$ for $\delta_{s}$ ), but also provides a natural non-linear fall-off away from this direction. Each term is also enhanced independently with individual scaling magnitudes $\gamma_{a}, \gamma_{d}$ and $\gamma_{s}$.

Figure 6-a shows results obtained with the scaled Phong Shading model using a single directional light (performances are reported inside the Figure). With such a minimal illumination, the depiction of curvature anisotropy becomes much more sensible; we thus usually make use of low $\lambda$ values in these settings. Scaling the ambient term gives results equivalent to mean-curvature shading [16] (see Figure 6b). Our method is also easily applied to Toon Shading: one only has to quantize the scaled reflected intensity. However, 


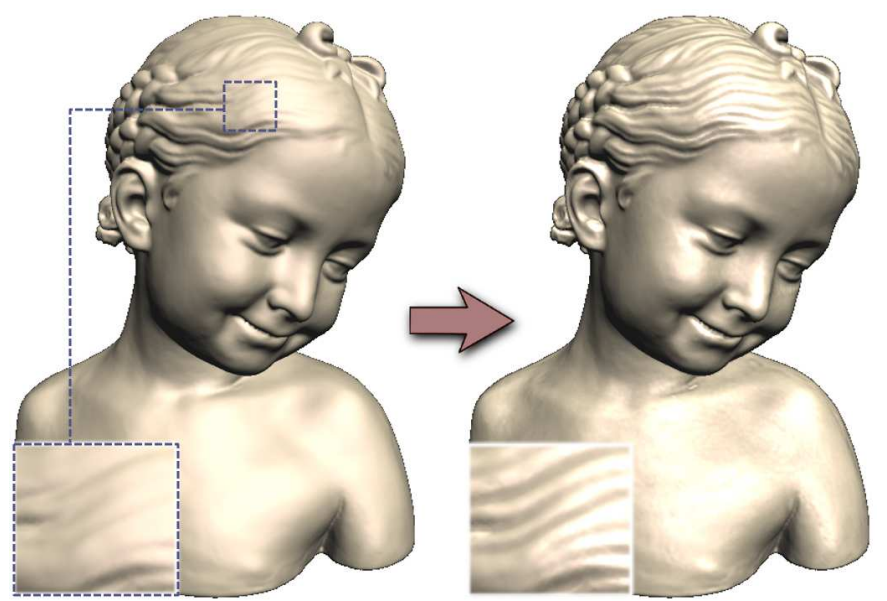

(a) $96 \mathrm{fps} / 384,266$ polygons

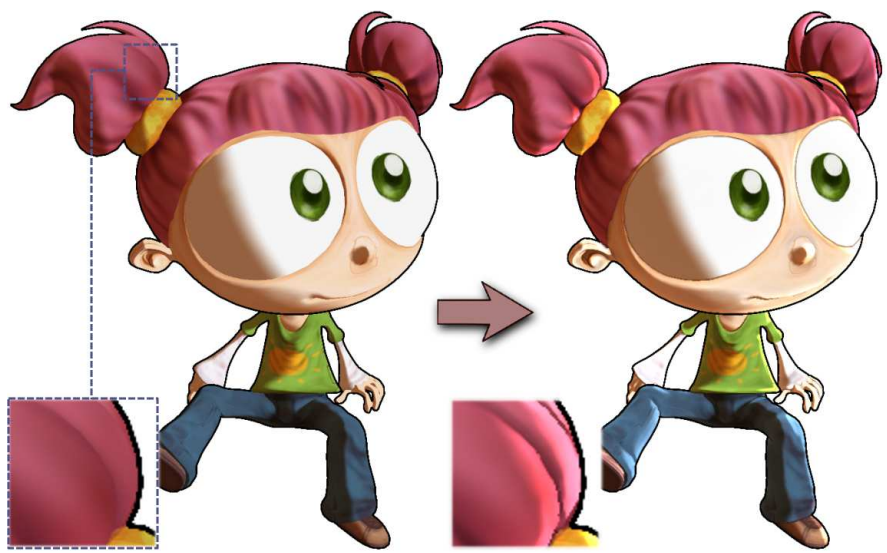

(c) $241 \mathrm{fps} / 48,532$ polygons

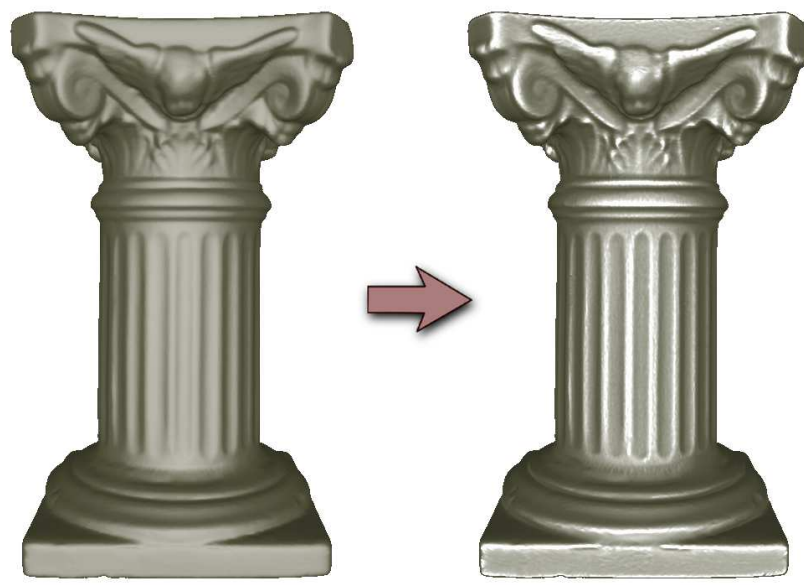

(b) $63 \mathrm{fps} / 2,101,000$ polygons

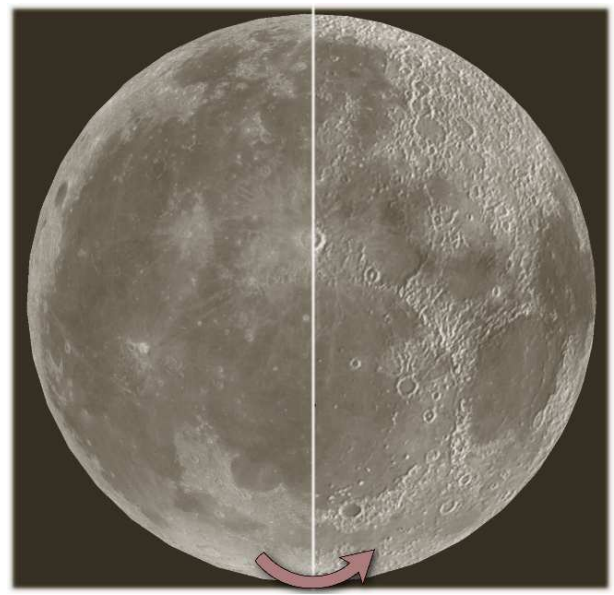

(d) $300 \mathrm{fps} / 1,600$ polygons

Fig. 6: Radiance Scaling in simple lighting scenarios: (a) Each lobe of Phong's shading model is scaled independently to reveal shape features such as details in the hair. (b) Radiance Scaling is equivalent to Mean Curvature Shading when applied to an ambient lobe (we combine it with diffuse shading in this Figure). (c) Surface features are also convincingly enhanced with Cartoon Shading, as with this little girl character (e.g., observe the right leg, the ear, the bunches, or the region around the nose). (d) Radiance Scaling is efficient even with sub-Lambertian materials, as in this example of a moon modeled by a sphere and a detailed normal map.

this quantization tends to mask subtle shading variations, and hence the effectiveness of Radiance Scaling is a bit reduced in this case. Nevertheless, as shown in Figure 6c, many surface details are still properly enhanced by the technique. We also applied our method to objects made of sub-Lambertian materials $\left(\rho_{s l}\left(\boldsymbol{\ell}_{0}\right)=\left(\mathbf{n} \cdot \boldsymbol{\ell}_{o}\right)^{\zeta}, \zeta \in[0,1)\right.$, with $\delta_{s l}=\rho_{s l}$ ). Figure 6-d illustrates this process with a sub-Lambertian moon $(\zeta=0.5)$ modeled as a smooth sphere with a detailed normal map.

To test our method in a video game context, we implemented an optimized version of Radiance Scaling using a single light source and Phong shading, and measured an overhead of 0.17 milliseconds per frame in $1024 \times 768$. Note that our technique is output-sensitive, hence this overhead is independent of scene complexity.

\subsection{Complex lighting with Ashikhmin-Shirley BRDF model}

Rendering in complex lighting environments with accurate material models may be done in a variety of ways. In our experiments, we evaluate Ashikhmin-Shirley BRDF model [21] using a dense sampling of directions at each surface point. As for Phong shading, we introduce reflectance mapping functions that let users control the enhancement of different shading terms independently.

Using $N$ light sources and Ashikmin-Shirley BRDF, Equation 1 becomes

$$
\begin{aligned}
L^{\prime}(\mathbf{p} \rightarrow \mathbf{e})= & \sum_{i=1}^{N} \rho_{d}\left(\boldsymbol{\ell}_{i}\right) \sigma_{d}\left(\mathbf{p}, \boldsymbol{\ell}_{i}\right) L\left(\boldsymbol{\ell}_{i}\right)+ \\
& \sum_{i=1}^{N} \rho_{s}\left(\mathbf{e}, \boldsymbol{\ell}_{i}\right) \sigma_{s}\left(\mathbf{p}, \mathbf{e}, \boldsymbol{\ell}_{i}\right) L\left(\boldsymbol{\ell}_{i}\right)
\end{aligned}
$$




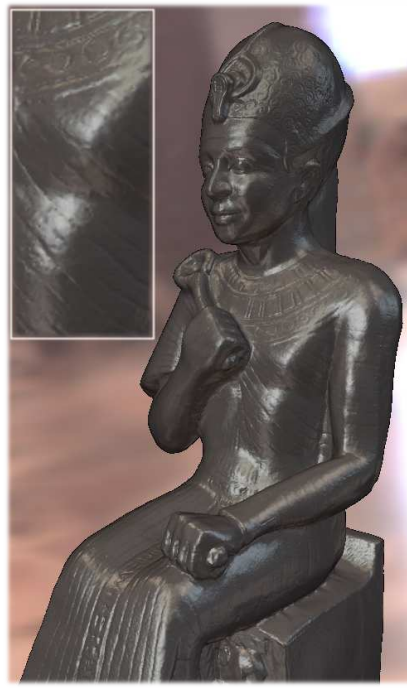

(a) without RS

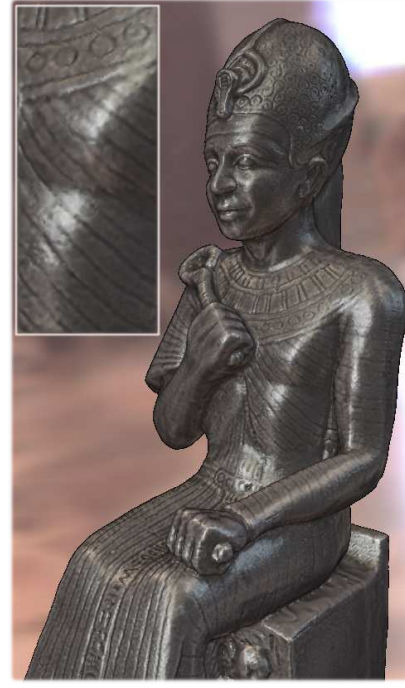

(b) RS applied to diffuse

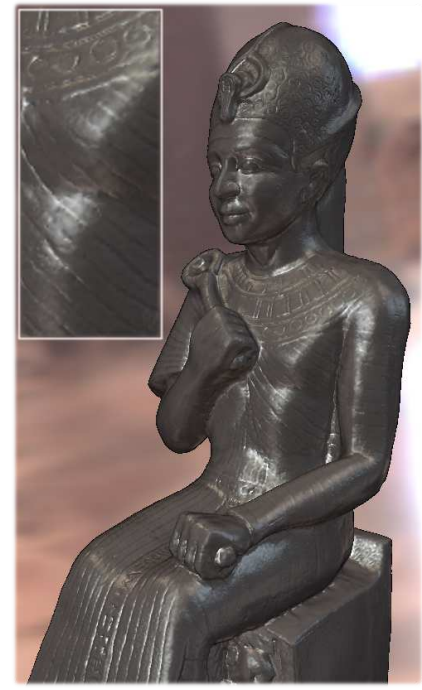

(c) RS applied to specular

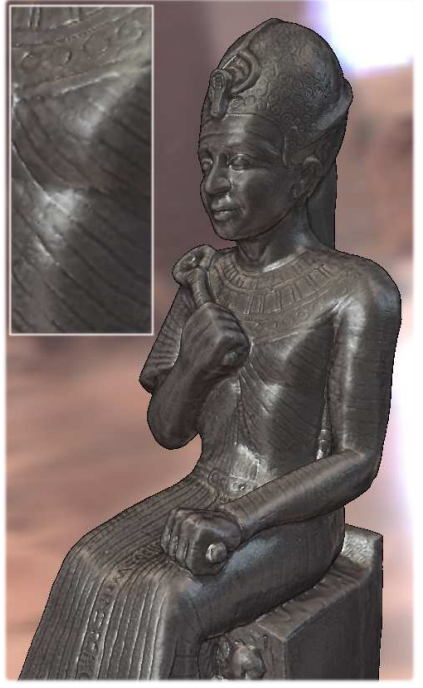

(d) RS applied to both

Fig. 7: Radiance Scaling using complex lighting: (a) A glossy object obtained with Ashikmin's BRDF model, with a zoomed view on the chest. (b) Applying Radiance Scaling only to the diffuse term mostly enhances surface features away from highlights (e.g., it darkens concave stripes on the arms and chest). (c) Applying it only to the specular term enhances surface features in a different way (e.g., it brightens some of the concave stripes, and enhances foreshortened areas). (d) Combining both enhancements brings up all surface details in a rich way (e.g., observe the alternations of bright and dark patterns on the chest).

where $\boldsymbol{\ell}_{i}$ is the $i$-th light source direction at point $\mathbf{p}$ and $\rho_{d}$ and $\rho_{s}$ correspond to the diffuse and specular lobes of Ashikhmin-Shirley BRDF model (see [21]).

As opposed to Phong's model, the diffuse and specular lobes of Ashikmin-Shirley BRDF model may be outside of the $[0,1]$ range, hence they cannot be used directly as mapping functions. Our alternative is to rely on each lobe's reference direction to compute reflectance mapping functions. We thus choose $\delta_{d}\left(\boldsymbol{\ell}_{i}\right)=\left(\boldsymbol{\ell}_{i} \cdot \mathbf{n}\right)$ for the diffuse term and $\delta_{s}\left(\mathbf{e}, \boldsymbol{\ell}_{i}\right)=\left(\mathbf{h}_{i} \cdot \mathbf{n}\right)$ for the specular term, where $\mathbf{h}_{i}$ is the half vector between $\boldsymbol{\ell}_{i}$ and the view direction e. As before, each term is enhanced with separate scaling magnitudes $\gamma_{d}$ and $\gamma_{s}$.

Figure 7 illustrates the use of Radiance Scaling on a glossy object with Ashikmin-Shirley model and an environment map (performances are reported in Section 6.1). First, the diffuse component is enhanced as shown in Figure 7-b: observe how concavities are darkened on the chest, the arms, the robe and the hat. The statue's face gives here a good illustration of how shading variations are introduced: the shape of the eyes, mouth and forehead wrinkles is more apparent because close concavities and convexities give rise to contrasted diffuse gradients. Second, the specular component is enhanced as shown in Figure 7-c: this makes the inscriptions on the robe more apparent, and enhances most of the details on the chest and the hat. Combining both enhanced components has shown in Figure 7-d produces a crisp depiction of surface details, while at the same time conserving the overall object appearance.

\subsection{Precomputed radiance data}

Global illumination techniques are usually time-consuming processes. For this reason, various methods have been proposed to precompute and reuse radiance data. Radiance Scaling introduces an additional term, $\sigma$, to the reflected radiance equation (see Equation 1). In the general case $\sigma$ depends both on a curvature mapping function $\kappa(\mathbf{p})$ and a reflectance mapping function $\delta(\mathbf{e}, \ell)$, which means that precomputing enhanced radiance data would require at least an additional storage dimension.

To avoid additional storage, we replace the general reflectance mapping function $\delta(\mathbf{e}, \boldsymbol{\ell})$ by a simplified one $\bar{\delta}(\mathbf{e})$ that is independent of lighting direction $\ell$. The scaling function $\sigma_{\alpha, \gamma}(\kappa(\mathbf{p}), \delta(\mathbf{e}, \boldsymbol{\ell}))$ is then replaced by a simplified version $\bar{\sigma}_{\alpha, \gamma}(\kappa(\mathbf{p}), \bar{\delta}(\mathbf{e}))$, noted $\bar{\sigma}(\mathbf{p}, \mathbf{e})$ and taken out of the integral in Equation 1:

$$
L^{\prime}(\mathbf{p} \rightarrow \mathbf{e})=\bar{\sigma}(\mathbf{p}, \mathbf{e}) \int_{\Omega} \rho(\mathbf{e}, \boldsymbol{\ell})(\mathbf{n} \cdot \boldsymbol{\ell}) L(\mathbf{p} \leftarrow \boldsymbol{\ell}) d \boldsymbol{\ell}
$$

Now the integral may be precomputed, and the result scaled. Even if scaling is not performed per incoming light direction anymore, it does depend on the curvature mapping function $\kappa$, and diffuse and specular components may be manipulated separately by defining dedicated reflectance mapping functions $\bar{\delta}_{d}$ and $\bar{\delta}_{s}$. In Sections 5.3.1 and 5.3.2, we show examples of such functions for perfect diffuse, and perfect reflective/refractive materials respectively. The exact same reflectance mapping functions could be used with more complex precomputed radiance transfer methods. 


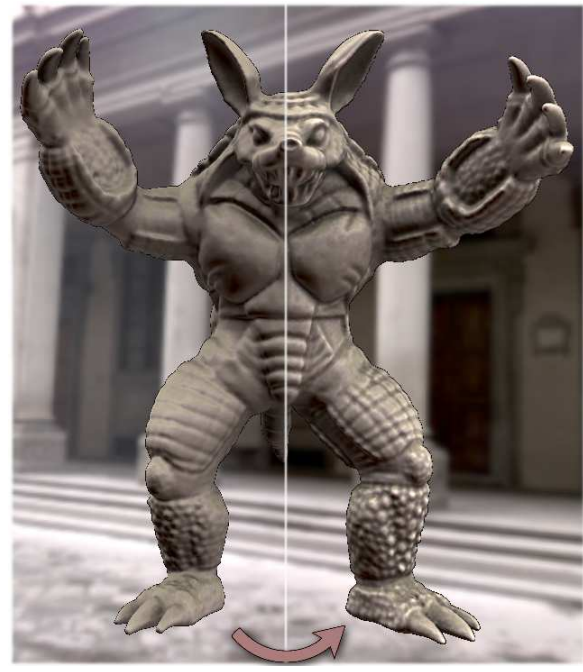

(a) RS applied to a prefiltered envmap

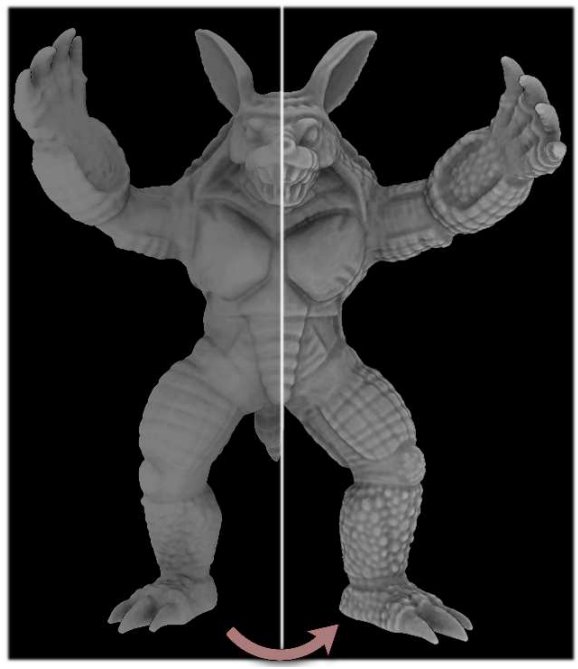

(b) RS applied to Ambient Occlusion

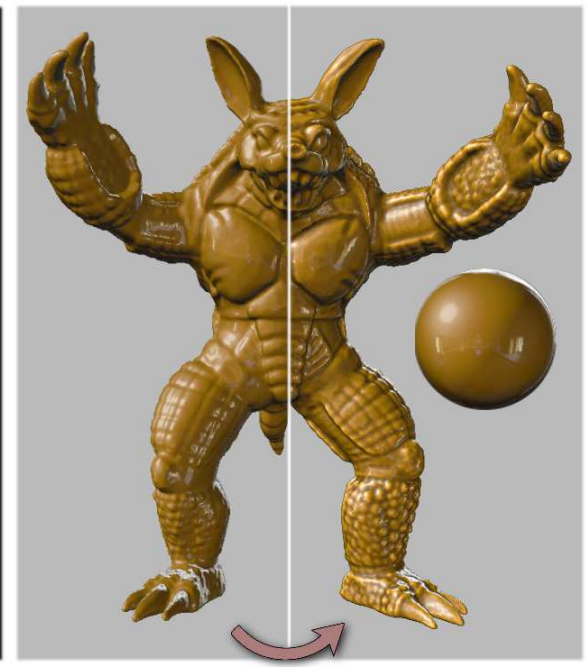

(c) RS applied to a Lit Sphere

Fig. 8: Radiance Scaling using precomputed lighting: (a) To improve run-time performance, precomputed radiance data may be stored in the form of ambient occlusion and prefiltered environment map. Radiance Scaling is easily adapted to such settings and provides enhancement at real-time frames rates (66 fps / 345, 944 polygons). (b) Even when only applied to the ambient occlusion term, Radiance Scaling produces convincing results. (c) For stylized rendering purposes, Radiance Scaling may be applied to a Lit Sphere rendering.

\subsubsection{Perfectly diffuse materials}

For diffuse materials, Ambient Occlusion [2] and Prefiltered Environment Maps [22] are among the most widely used techniques to precompute radiance data. In the following, we show a similar approximation used in conjunction with Radiance Scaling. The BRDF is first considered constant diffuse: $\rho(\mathbf{e}, \boldsymbol{\ell})=\rho_{d}$. We then consider only direct illumination from an environment map: $L(\mathbf{p} \leftarrow \boldsymbol{\ell})=$ $V(\boldsymbol{\ell}) L_{e n v}(\boldsymbol{\ell})$ where $V \in\{0,1\}$ is a visibility term and $L_{e n v}$ is the environment map. Equation 4 then becomes:

$$
L^{\prime}(\mathbf{p} \rightarrow \mathbf{e})=\bar{\sigma}(\mathbf{p}, \mathbf{e}) \rho_{d} \int_{\Omega}(\mathbf{n} \cdot \boldsymbol{\ell}) V(\boldsymbol{\ell}) L_{e n v}(\boldsymbol{\ell}) d \boldsymbol{\ell}
$$

We then approximate the enhanced radiance with

$$
L^{\prime}(\mathbf{p} \rightarrow \mathbf{e}) \simeq \bar{\sigma}(\mathbf{p}, \mathbf{e}) \rho_{d} A(p) \bar{L}(\mathbf{n})
$$

with $A(p)$ the ambient occlusion stored at each vertex, and $\bar{L}$ an irradiance average stored in a prefiltered environment map:

$$
A(\mathbf{p})=\int_{\Omega}(\mathbf{n} \cdot \boldsymbol{\ell}) V(\boldsymbol{\ell}) d \boldsymbol{\ell}, \quad \bar{L}(\mathbf{n})=\int_{\Omega} L_{e n v}(\boldsymbol{\ell}) d \boldsymbol{\ell} .
$$

For perfectly diffuse materials, we use the reflectance mapping function $\bar{\delta}_{d}(\mathbf{p})=\bar{L}(\mathbf{n}) / \bar{L}^{*}$, with $\mathbf{n}$ the normal at $\mathbf{p}$, and $\bar{L}^{*}=\max _{\mathbf{n}} \bar{L}(\mathbf{n})$ the maximum averaged radiance found in the prefiltered environment map. This choice is coherent with perfectly diffuse materials since in this case the light direction that contributes the most to reflected light intensity is the normal direction on average.

Figure 8-a shows the warping of prefiltered environment maps using the Armadillo model. Observe how macrogeometry patterns are enhanced on the leg, arm and forehead. The ambient occlusion term is shown separately in

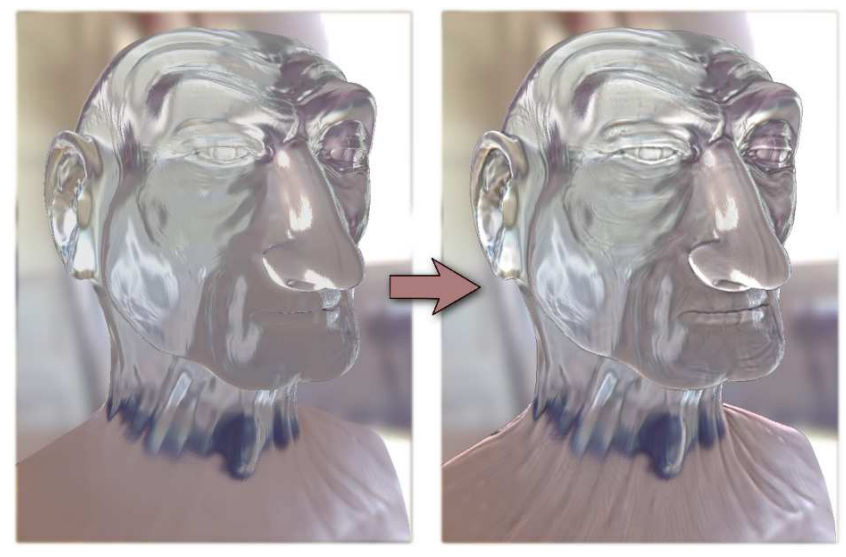

Fig. 9: Radiance Scaling with refraction: Even with a refractive material (here we use a simple one-bounce approximation), Radiance Scaling is able to convey surface shape effectively as in Figure 1.

Figure 8-b. An alternative to using a prefiltered environment map for stylized rendering purpose is the Lit Sphere [23]. It consists in a painted sphere where material, style, and illumination direction are implicitly given, and has been used for volumetric rendering [24] and in the ZBrush ${ }^{\circledR}$ software (under the name "matcap"). Radiance Scaling produces convincing results with Lit Spheres as shown in Figure 8-c.

\subsubsection{Perfectly reflective and refractive materials}

The case of perfectly reflective or refractive materials is quite similar to the perfectly diffuse one. If we consider a perfectly reflective/refractive material $\rho_{s}$ (a dirac in the 


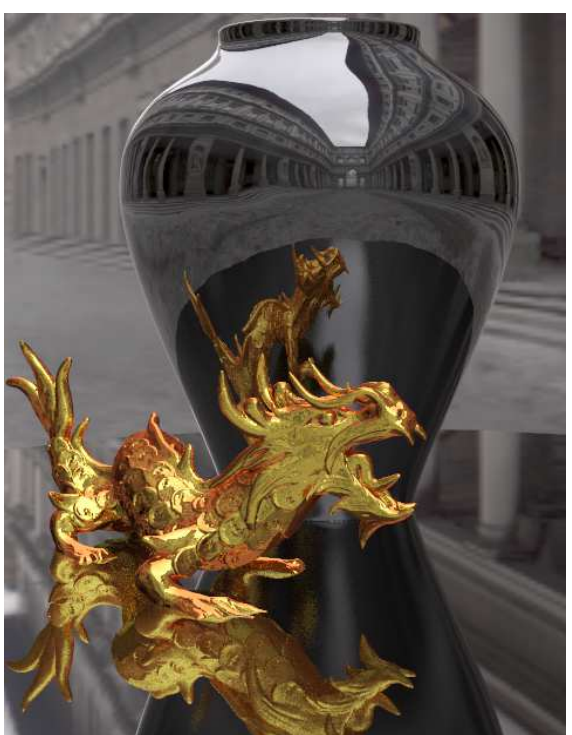

(a) without RS

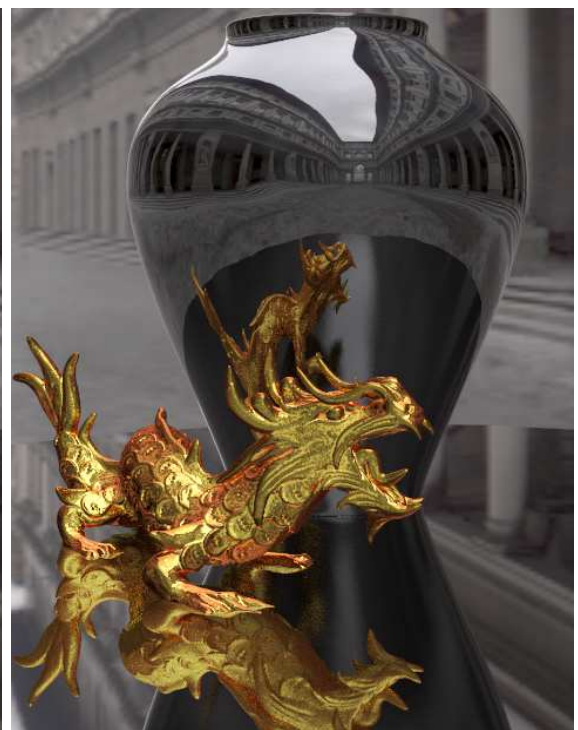

(b) RS applied to first bounce

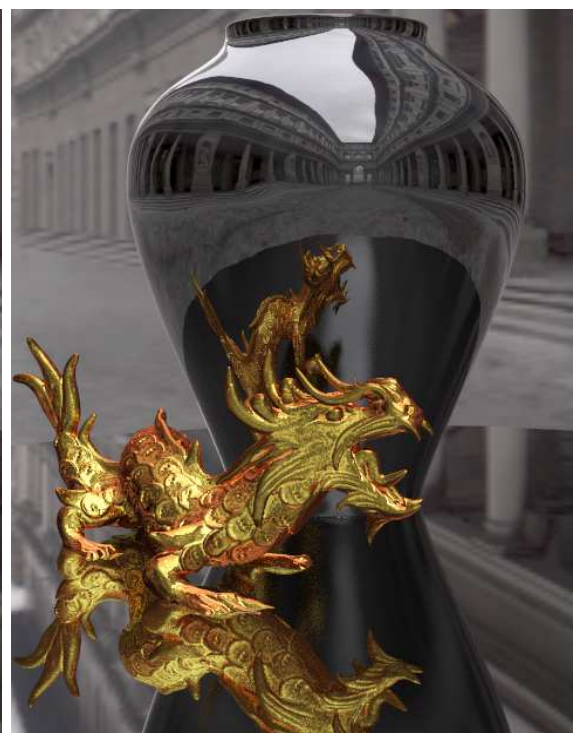

(c) RS applied to all bounces

Fig. 10: Radiance Scaling with inter-reflections: (a) A 3D scene composed of a dragon model with a shiny material, and a vase on a plane, both with a mirror material. Some details on the dragon itself and on its reflections are hard to distinguish. (b) Radiance Scaling applied to the first ray bounce enhances the dragon surface itself (its scales in particular), but not its reflections. (c) Radiance Scaling applied to all ray bounces enhances its surface in reflections as well (observe its scales again).

reflected/refracted direction $\mathbf{r}$ ) and ignore the visibility term, then Equation 4 becomes:

$$
L^{\prime}(\mathbf{p} \rightarrow \mathbf{e})=\bar{\sigma}(\mathbf{p}, \mathbf{e}) L_{e n v}(\mathbf{r})
$$

We use the reflectance mapping function $\bar{\delta}_{s}(\mathbf{e})=$ $L_{e n v}(\mathbf{r}) / L_{e n v}^{*}$, with $\mathbf{r}$ the reflected/refracted view direction and $L_{e n v}^{*}=\max _{\mathbf{r}} L_{e n v}(\mathbf{r})$ the maximum irradiance in the environment map. This choice is coherent with perfectly reflective/refractive materials, since in this case the light direction that contributes the most to reflected light intensity is the reflected/refracted view direction.

Figure 9 shows how Radiance Scaling enhances surface features with a simple approximation of a purely refractive material. With a very similar approach, it is also able to enhance mirror-like materials, using the reflected view direction instead of the refracted one.

\subsection{Inter-reflections and soft shadows}

For more complex renderings involving global illumination, we resort to Equation 1 and use the reflectance mapping function of Section 5.2. Inter-reflections are illustrated in Figure 10 on a scene composed of objects with shiny materials using Ashikhmin-Shirley BRDF (as in Section 5.2). As with previous examples, we could enhance the first bounce only as seen in the middle image. However, the same mechanism is easily applied to incoming radiance, and hence to all the subsequent ray bounces as illustrated in the right image. Here we have used an object-space curvature measure for simplicity. Note how details such as dragon scales are better perceived in both direct viewing

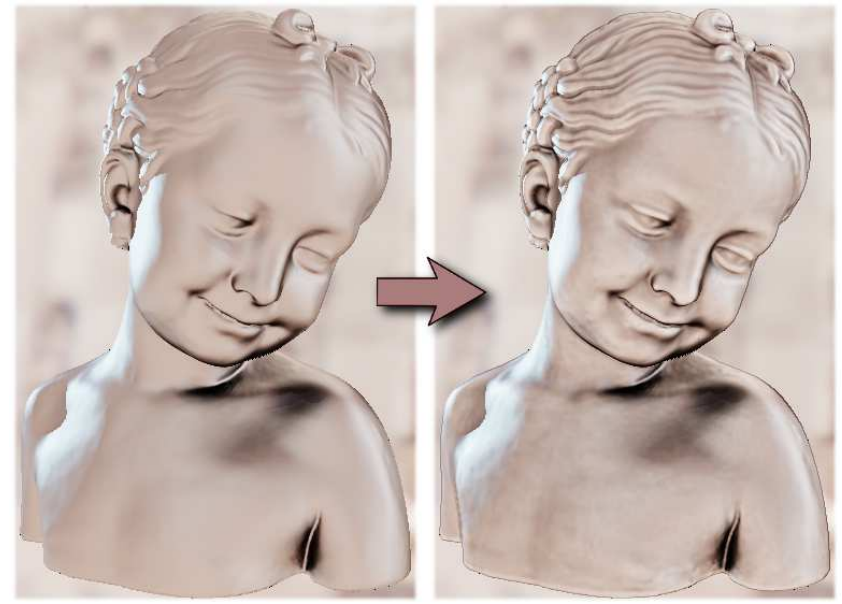

Fig. 11: Radiance Scaling with area lights: Area lights are densely sampled in this rendering, with visibility computed per light ray and leading to soft shadows. When applied in this configuration, Radiance Scaling preserves soft shadow shape, but still enhances surface details appropriately.

and reflections. Soft-shadows are illustrated in Figure 11: Radiance Scaling is applied to a simple scene where arealights are densely samples and visibility is computed per light sample. The shape of the soft shadow is not altered by enhancement, and yet many surface details are conveyed. With these global illumination scenarios, the use of Radiance Scaling (both applied to first and all bounces) is negligible: the overhead is smaller than a second for the images of Figure 10 that take around 10 minutes to render in our implementation. 


\section{Discussion}

\subsection{Comparisons with previous work}

Our approach is designed to depict local surface features, and is difficult to compare with approaches such as Accessibility Shading that consider more of the surrounding geometry. Accessibility Shading characterizes how easily a surface may be touched by a spherical probe, and thus tends to depict more volumetric features. However, for surfaces where small-scale relief dominates large-scale variations (such as carved stones or roughly textured statues), the spherical probe acts as a curvature measure. In this case, Accessibility Shading becomes similar to Mean Curvature Shading, which is a special case of Radiance Scaling as seen in Figure 6-b.

A technique related to Accessibility Shading is Ambient Occlusion: indeed, measuring occlusion from visible geometry around a surface point is another way of probing a surface. Ambient Occlusion is more efficient at depicting proximity relations between objects (such as contacts), and deep cavities. However, as seen in Figure 8-b, it also misses shallow (yet salient) surface details, or even smoothes them out. Radiance Scaling reintroduces these details seamlessly. Both methods are thus naturally combined to depict different aspects of object shape.

The Polynomial Texture Maps (PTM) technique [25] introduces enhancement abilities similar to ours in the context of diffuse shading. However, the method modifies the diffuse lobe by a uniform gain that does not take surface features into account. In future work, we plan to apply Radiance Scaling to PTMs using normals estimated from PTMs themselves. Although the quality of enhancement will depend on the quality of the normal map, we believe it will bring more accurate enhancement abilities to PTMs.

3D Unsharp Masking provides yet another mean to enhance shape features: by enhancing outgoing radiance with a Cornsweet illusion effect, object shape properties correlated to shading are enhanced along the way. Besides the fact that users have little control on what property of a scene will be enhanced, 3D Unsharp Masking tends to make flat surfaces appear rounded, as in Cignoni et al. [17]. It is also limited regarding material appearance, as pointed out in Vergne et al. [4]. We thus focus on a comparison with Light Warping in the remainder of this Section.

An important advantage of Radiance Scaling over Light Warping is that it does not require a dense sampling of the environment illumination, and thus works in simple rendering settings as described in Section 5.1. As an example, consider Toon Shading. Light Warping does allow to create enhanced cartoon renderings, but for this purpose makes use of a minimal environment illumination, and still requires to shoot multiple light rays. Radiance Scaling avoids such unnecessary sampling of the environment as it works with a single light source. Hence it is much faster to render: the character in Figure 6 is rendered at $241 \mathrm{fps}$ with Radiance

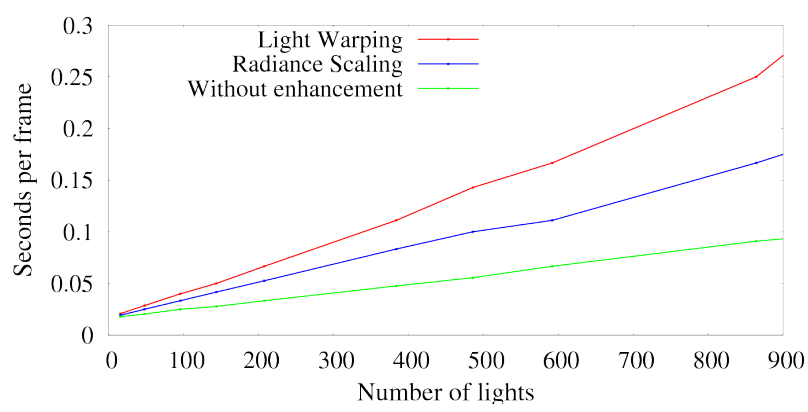

Fig. 12: This plot gives the performances obtained with the scene shown in Figure 7 without enhancement, and with both Radiance Scaling and Light Warping. The 3D model is composed of 1,652,528 polygons. While the time for rendering a single frame increases linearly with the number of light samples in all cases, our novel method is linearly faster than Light Warping.

Scaling, whereas performances drop to $90 \mathrm{fps}$ with Light Warping as it requires at least 16 illumination samples to give a convincing result.

For more complex materials, Radiance Scaling is also faster than Light Warping, as seen in Figure 12. However, the two methods are not qualitatively equivalent, as shown in Figure 13. For diffuse materials and with natural illumination, the two methods produce similar results: concavities are depicted with darker colors, and convexities with brighter colors. However, for some orientations of the viewpoint relative to the environment illumination, Light Warping may reverse this effect, since rays are attracted toward or away from the camera regardless of light source locations. Radiance Scaling does not reverse tone in this manner. The main difference between the two techniques appears with shiny materials. In this case, the effect of enhancement on illumination is more clearly visible: Light Warping modulates lighting frequency whereas Radiance Scaling modulates lighting intensity.

We have also tried combining the two methods together, and have found that dual enhancement is most effective with glossy materials, as observed in Figure 14. In this case, Light Warping is efficient at deforming reflected light patterns in such a way that they better separate convexities and concavities, but it relies on the presence of lighting variations in the environment, and as a result may not be able to enhance all details. In contrast, Radiance Scaling does not rely on lighting variations but rather modulates existing shading. Combining both methods produces a more compelling result where all the details are enhanced and convexities \& concavities are easily distinguished from each other.

\subsection{Directions for future work}

We have shown that the adjustment of reflected light intensities, a process we call Radiance Scaling, provides 

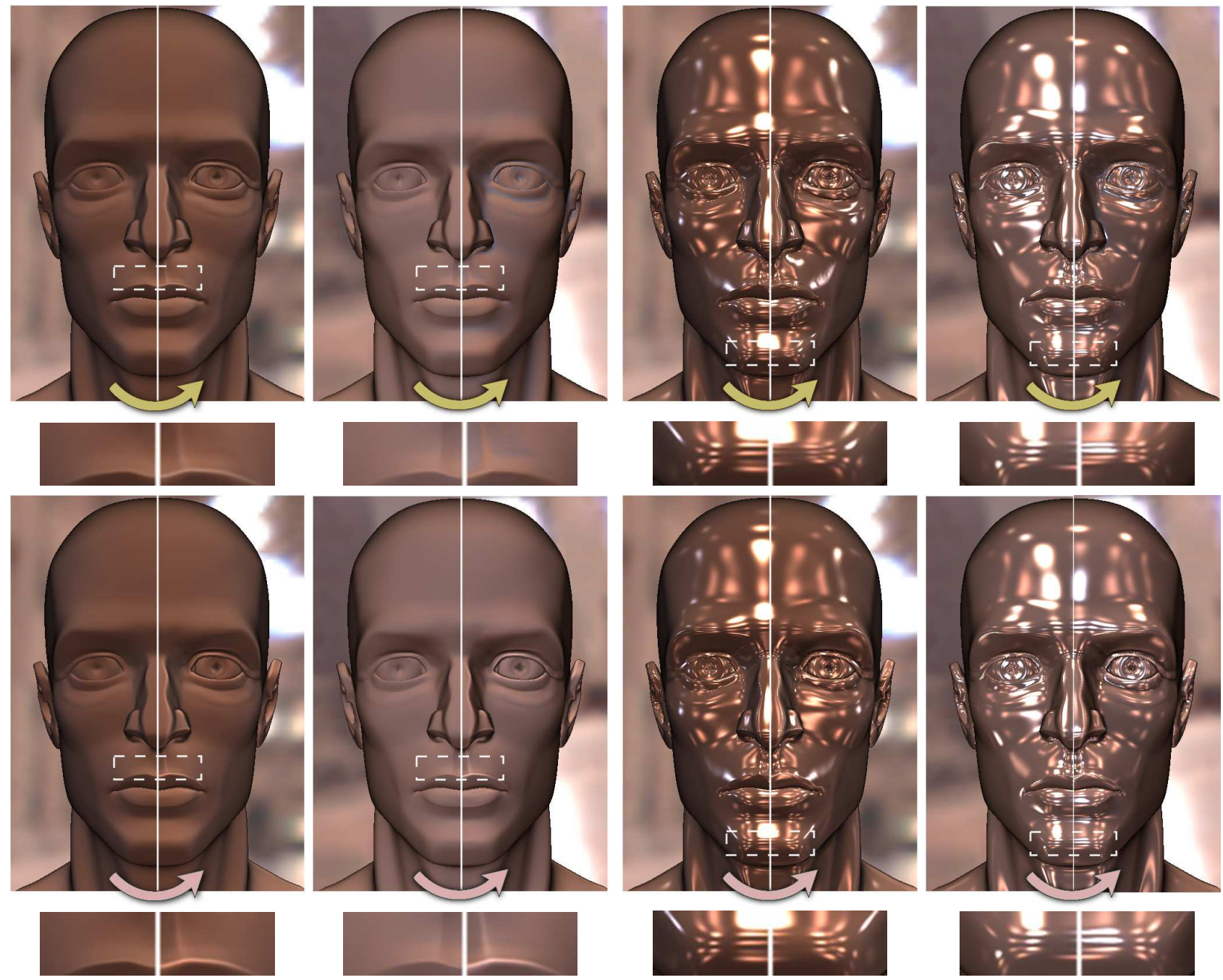

(a)

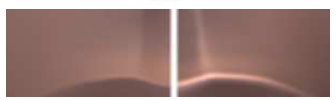

(b)

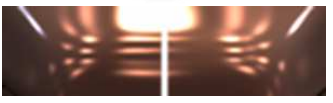

(c)

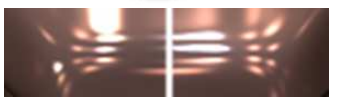

(d)

Fig. 13: Comparison with Light Warping. Top row: Light warping image. Bottom row: Radiance Scaling. (a-b) Both methods show similar enhancement abilities when used with a diffuse material and a natural illumination environment: convexities exhibit brighter colors, and concavities darker colors in most cases. For some orientation of the viewpoint relative to the environment, Light Warping may reverse this effect though (concavities are brighter, convexities darker) whereas Radiance Scaling does not. (c-d) The methods are most different with shiny objects, shown with two illumination orientations as well.

a versatile approach to the enhancement of surface shape through shading. However, when the enhancement magnitude is pushed to extreme values, our method alters material appearance. This is because variations in shape tend to dominate variations due to shading. An exciting avenue of future work would be to characterize perceptual cues to material appearance and preserve them through enhancement.

Although Radiance Scaling produces convincing enhancement in many rendering scenarios, there is still room for alternative enhancement techniques. Indeed, our approach makes two assumptions that could be dropped in future work: 1) concave and convex features have inverse effects on scaling; and 2) enhancement is obtained by local differential operators. The class of reflected lighting patterns humans are able to make use for perceiving shape is obviously much more diverse than simple alternations of bright and dark colors in convexities and concavities [26]. And these patterns are likely to be dependent on the main illumination direction (e.g., [27], [28], [29]), material characteristics (e.g., [30], [31]), motion (e.g., [32], [33]), and silhouette shape (e.g., [34]). Characterizing such patterns is a challenging avenue of future work.

\section{ACKNOWLEDGMENTS}

We thank TeamTo for providing us with the little girl model in Figure 6-c (ANGELO RULES 2009 - TeamTO - Cake Entertainment - francetélévisions - TéléTOON - Expand Drama), the Aim@Shape library for the 3D models of 


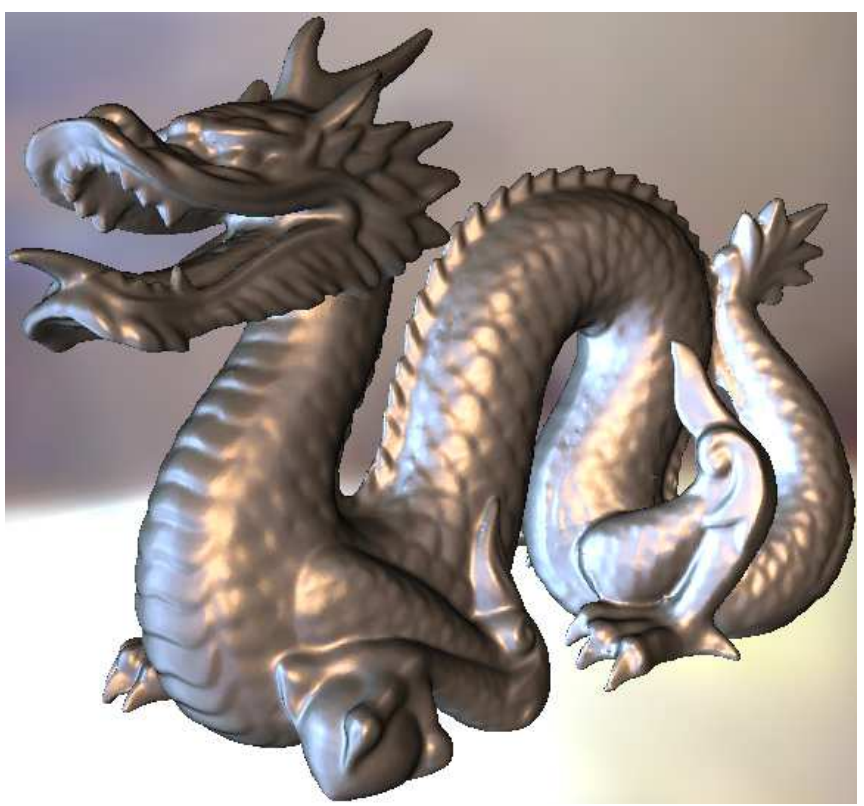

(a) Without enhancement

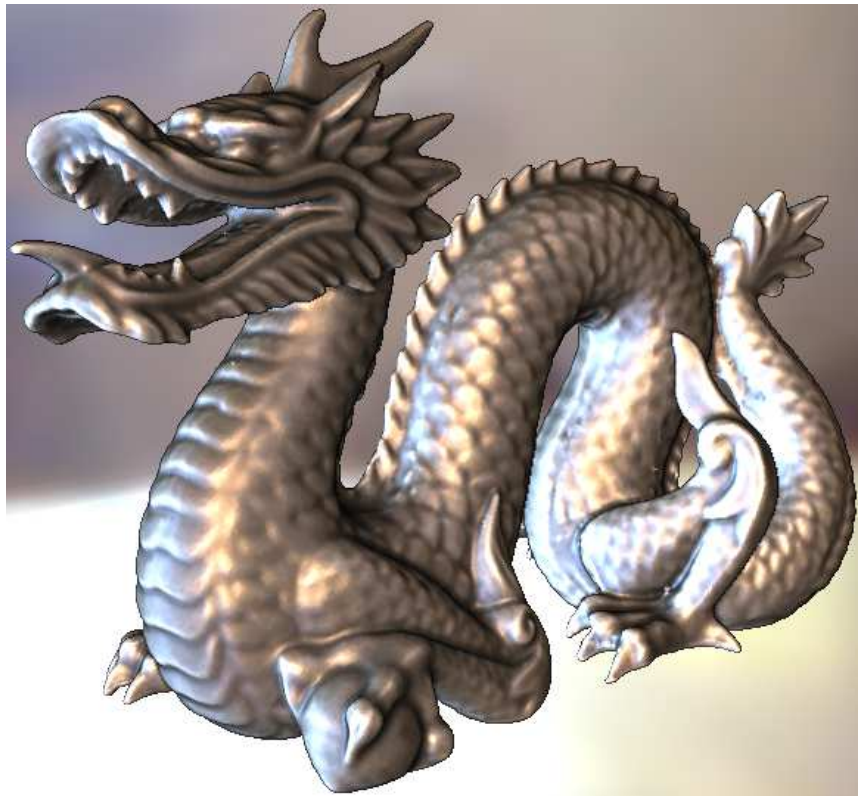

(c) With Radiance Scaling

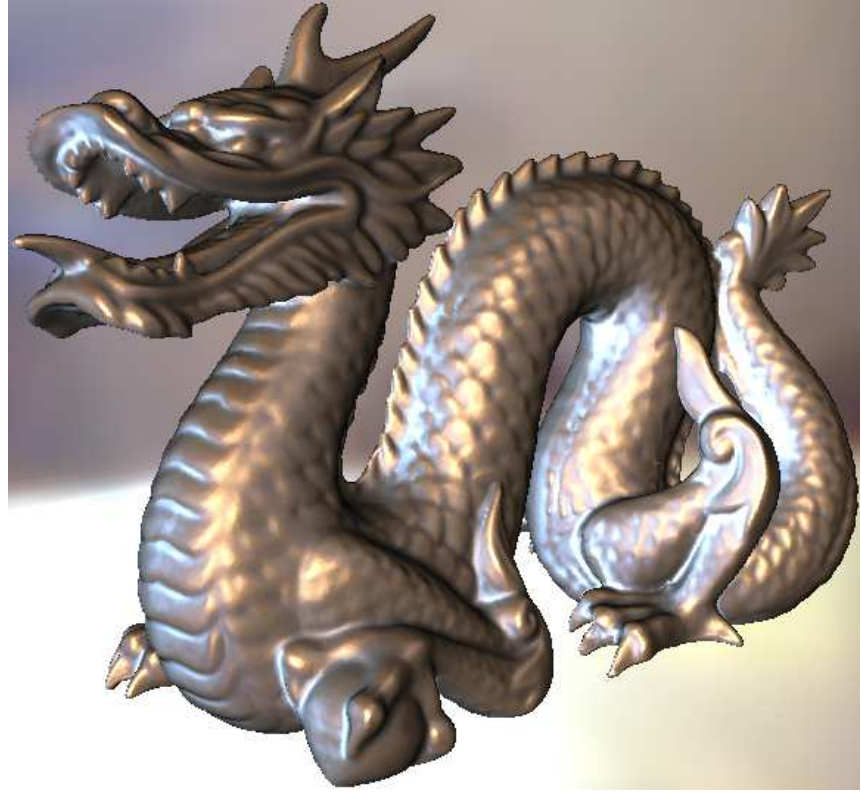

(b) with Light Warping

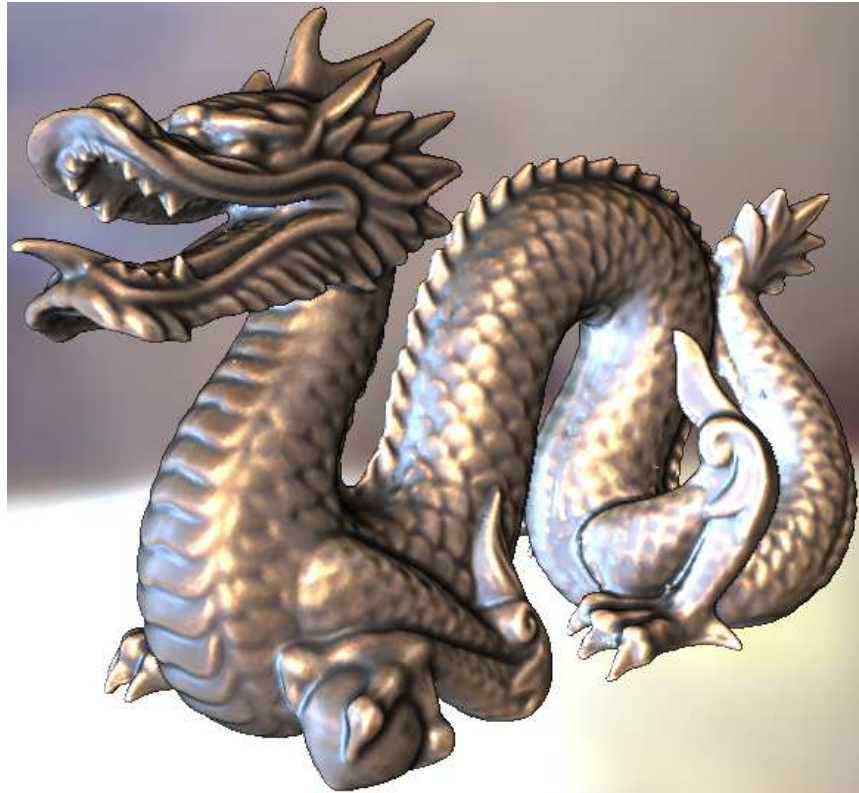

(d) with Light Warping and Radiance Scaling

Fig. 14: Combining Radiance Scaling with Light Warping: (a) The Chinese dragon model rendered with a glossy material without enhancement. (b) Light Warping concentrates backlighting in concavities and front lighting in convexities, hence making surface shape more comprehensible (e.g., on the neck). (c) Radiance Scaling brings out more surface details, but without conpressing or stretching reflected patterns (e.g., on the face). (d) Combining both techniques produces a more compelling enhancement: convexities and concavities are better segregated and their contrast is increased.

Figure 6-a and 7, and Paul Debevec for the environment maps used throughout the paper. This work has been sponsored by the ANR Animare (ANR-08-JCJC-0078-01) and SeARCH (ANR-09-CORD-019) projects. Xavier Granier is supported by the Open Project Program of the State Key Lab of CAD\&CG (Grant No. A1007), Zhejiang University.

\section{REFERENCES}

[1] T. Saito and T. Takahashi, "Comprehensible Rendering of 3-D Shapes," in Proc. ACM SIGGRAPH '90. ACM, 1990, pp. 197206.

[2] M. Pharr and S. Green, GPU Gems. Addison-Wesley, 2004, ch. Ambient Occlusion

[3] T. Ritschel, K. Smith, M. Ihrke, T. Grosch, K. Myszkowski, and H.P. Seidel, "3D Unsharp Masking for Scene Coherent Enhancement," ACM Trans. Graph. (Proc. SIGGRAPH 2008), vol. 27, no. 3, pp. $1-8,2008$ 
[4] R. Vergne, R. Pacanowski, P. Barla, X. Granier, and C. Schlick, "Light warping for enhanced surface depiction," ACM Transaction on Graphics (Proceedings of SIGGRAPH 2009), Aug 2009.

[5] _ - "Radiance scaling for versatile surface enhancement," $I 3 D$ ' 10 : Proc. symposium on Interactive 3D graphics and games, Feb 2010

[6] M. Nienhaus and J. Döllner, "Blueprints: illustrating architecture and technical parts using hardware-accelerated non-photorealistic rendering," in Graphics Interface (GI'04). Canadian HumanComputer Communications Society, 2004, pp. 49-56.

[7] Y. Ohtake, A. Belyaev, and H.-P. Seidel, "Ridge-valley lines on meshes via implicit surface fitting," ACM Trans. Graph. (Proc. SIGGRAPH 2004), vol. 3, no. 23, pp. 609-612, 2004

[8] D. DeCarlo, A. Finkelstein, S. Rusinkiewicz, and A. Santella, "Suggestive Contours for Conveying Shape," ACM Trans. Graph. (Proc. SIGGRAPH 2003), vol. 22, no. 3, pp. 848-855, 2003.

[9] T. Judd, F. Durand, and E. H. Adelson, "Apparent Ridges for Line Drawing," ACM Trans. Graph. (Proc. SIGGRAPH 2007), vol. 26, no. 3, p. 19, 2007.

[10] Y. Lee, L. Markosian, S. Lee, and J. F. Hughes, "Line drawings via abstracted shading," ACM Trans. Graph., vol. 26, no. 3, p. 18, 2007.

[11] T. Goodwin, I. Vollick, and A. Hertzmann, "Isophote distance: a shading approach to artistic stroke thickness," in NPAR '07: Proc. international symposium on Non-photorealistic animation and rendering. ACM, 2007, pp. 53-62.

[12] M. Kolomenkin, I. Shimshoni, and A. Tal, "Demarcating Curves for Shape Illustration," ACM Trans. Graph. (Proc. SIGGRAPH Asia 2008), vol. 27 , no. 5 , pp. $1-9,2008$

[13] L. Zhang, Y. He, X. Xie, and W. Chen, "Laplacian Lines for Real Time Shape Illustration," in I3D '09: Proc. symposium on Interactive $3 D$ graphics and games. ACM, 2009.

[14] G. Miller, "Efficient algorithms for local and global accessibility shading," in SIGGRAPH '94: Proceedings of the 21st annual conference on Computer graphics and interactive techniques. ACM, 1994, pp. 319-326.

[15] T. Cornsweet, Visual Perception. New York: Academic Press, 1970

[16] G. Kindlmann, R. Whitaker, T. Tasdizen, and T. Möller, "CurvatureBased Transfer Functions for Direct Volume Rendering: Methods and Applications," in Proc. IEEE Visualization 2003, October 2003 , pp. 513-520.

[17] P. Cignoni, R. Scopigno, and M. Tarini, "A simple Normal Enhancement technique for Interactive Non-photorealistic Renderings," Comp. \& Graph., vol. 29, no. 1, pp. 125-133, 2005.

[18] S. Rusinkiewicz, M. Burns, and D. DeCarlo, "Exaggerated Shading for Depicting Shape and Detail," ACM Trans. Graph. (Proc. SIGGRAPH 2006), vol. 25, no. 3, pp. 1199-1205, 2006.

[19] R. Vergne, P. Barla, X. Granier, and C. Schlick, "Apparent relief: a shape descriptor for stylized shading," in NPAR '08: Proc. international symposium on Non-photorealistic animation and rendering. ACM, 2008, pp. 23-29.

[20] G. Cipriano, G. N. P. Jr., and M. Gleicher, "Multi-scale surface descriptors," IEEE Trans. Vis. Comput. Graph., vol. 15, no. 6, pp. 1201-1208, 2009.

[21] M. Ashikhmin, S. Premoze, and P. Shirley, "A microfacet-based BRDF generator," in Proc. ACM SIGGRAPH '00. ACM, 2000, pp. 65-74.

[22] J. Kautz, P.-P. Vázquez, W. Heidrich, and H.-P. Seidel, "Unified Approach to Prefiltered Environment Maps," in Proceedings of the Eurographics Workshop on Rendering Techniques 2000. SpringerVerlag, 2000, pp. 185-196.

[23] P.-P. J. Sloan, W. Martin, A. Gooch, and B. Gooch, "The lit sphere: A model for capturing NPR shading from art," in Graphics interface 2001. Canadian Information Processing Society, 2001, pp. 143-150.
[24] S. Bruckner and M. E. Gröller, "Style transfer functions for illustrative volume rendering," Computer Graphics Forum, vol. 26, no. 3, pp. 715-724, Sep. 2007.

[25] T. Malzbender, D. Gelb, and H. Wolters, "Polynomial texture maps," in SIGGRAPH '01: Proceedings of the 28th annual conference on Computer graphics and interactive techniques. ACM, 2001, pp. 519-528.

[26] D. A. v. Koenderink J.J., The visual neurosciences. MIT Press, Cambridge, 2003, ch. Shape and shading, pp. 1090-1105.

[27] Y.-X. Ho, M. S. Landy, and L. T. Maloney, "How direction of illumination affects visually perceived surface roughness," J. Vis., vol. 6, no. 5, pp. 634-648, 52006.

[28] F. Caniard and R. W. Fleming, "Distortion in 3D shape estimation with changes in illumination," in $A P G V$ '07: Proc. symposium on Applied perception in graphics and visualization. ACM, 2007, pp. 99-105.

[29] J. P. O'Shea, M. S. Banks, and M. Agrawala, "The assumed light direction for perceiving shape from shading," in APGV '08: Proc. symposium on Applied perception in graphics and visualization. ACM, 2008, pp. 135-142.

[30] E. H. Adelson, "On seeing stuff: the perception of materials by humans and machines," in Society of Photo-Optical Instrumentation Engineers (SPIE) Conference Series, ser. Presented at the Society of Photo-Optical Instrumentation Engineers (SPIE) Conference, B. E. Rogowitz and T. N. Pappas, Eds., vol. 4299, Jun. 2001, pp. 1-12.

[31] P. Vangorp, J. Laurijssen, and P. Dutré, "The influence of shape on the perception of material reflectance," ACM Trans. Graph. (Proc. SIGGRAPH 2007), vol. 26, no. 3, p. 77, 2007.

[32] K. J. Pont S.C., Computer Analysis of Images and Patterns. Springer, Berlin, 2003, ch. Illuminance flow, pp. 90-97.

[33] Y. Adato, Y. Vasilyev, O. Ben Shahar, and T. Zickler, "Toward a theory of shape from specular flow," in ICCV07, 2007, pp. 1-8.

[34] R. W. Fleming, A. Torralba, and E. H. Adelson, "Specular reflections and the perception of shape," J. Vis., vol. 4, no. 9, pp. 798-820, 9 2004. 